\section{CLASSIFICATION OF RHEOLOGICAL PROPERTIES}

$\mathrm{T}$ HROUGH the courtesy of Prof. M. L. Oliphant, the British Rheologists' Club held a meeting at the Physics Department, University of Birmingham, on Saturday, January 24. After business proceedings, a discussion on "Classification of Rheological Properties" was introduced by Dr. L. R. G. Treloar, on behalf of the committee of the Club.

Dr. Treloar reminded members that at the annual general meeting of the Club, the question of definitions had been discussed and the committee had been instructed to draw up a tentative scheme for classification of properties of materials subjected to specific conditions, rather than to define terms many of which might not even approximate to the behaviour of real materials. This scheme, which was to be regarded as that it is believed that real materials follow these definitions exactly. We need not suppose that any material shows a perfect yield-value; still less that there are any perfect Bingham systems in Nature (that is, systems which flow at a rate strictly pro. portional to the excess of the applied pressure over the yield-value). These categories are of use in giving a practical approximation to the behaviour of real materials.

Deformations which are produced only after a yield-value has been exceeded but which do not obey Bingham's law may be subdivided into inelastic and plasto-elastic; the latter differs from viscoelastic only in showing a yield-value.

Returning to viscous systems which show no yieldvalue, these may show Newtonian (rate of deformation proportional to stress) or non-Newtonian flow. The latter group subdivides into inelastic and visco. elastic, thus re-merging with the elastic group.

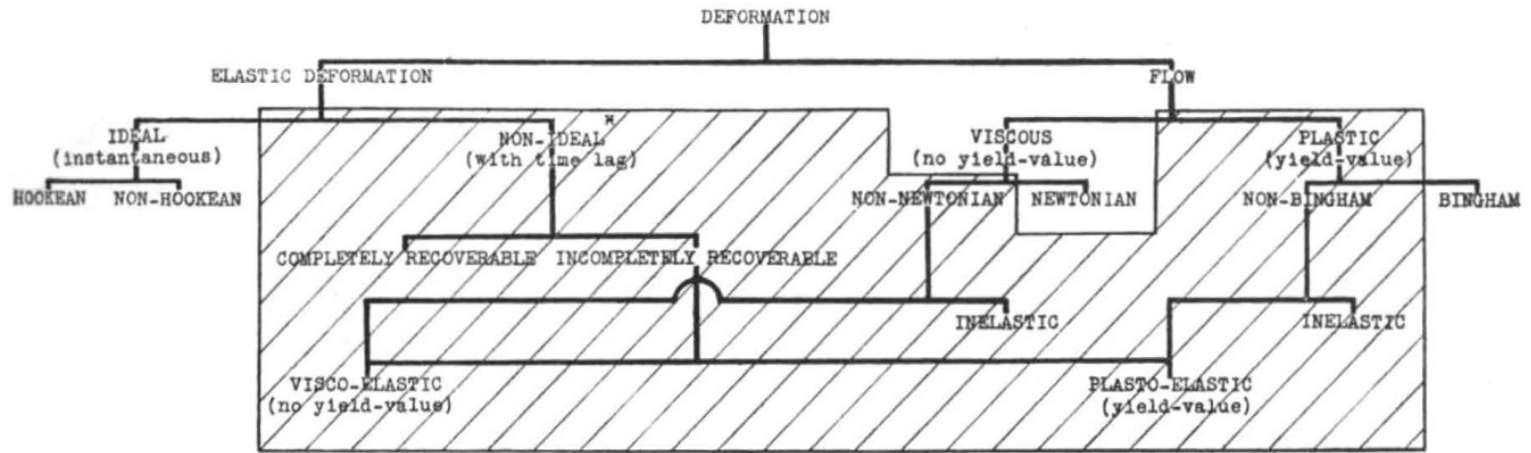

Elastic Deformation - recoverable deformation.

FLow - non-recoverable deformation.

When flow starts only above a critical streks (YIELD-VALUE) it is PLAsmc. When flow starts at all stresses, however small, it is Viscous.

HOOKFAN - obeys Hooke's law, stress proportional to strain.

\section{WORKING DEFINITIONS}

NEwTONYAN - obeys Newton's law, stress proportional to rate of shear. BINGHAM - obeys Bingham's law, rate of shear proportional to stress less yield-value.

An elastic deformation is IDEAI when the whole of it follows immediately on the application of a stress. When it does not, it is classed as NON-IDEAL.

NoTs. Categories within the hatched area are liable to change of rate of deformation under constant stress, the rate either increasing or decreasing.

* In the case of a spring and dash-pot in parallel, the system could obey Hooke's law if the stress were applied sufficiently slowly but not if it were applied fast. In some other systems a direct proportionality between stress and strain is not obtained however slowly the stress is applied.

preliminary and subject to modification, is shown on the accompanying table.

Deformations are conveniently subdivided into elastic deformations and flow, the former being generally defined as 'recoverable' and the latter as non-recoverable'. Recoverable deformations are subdivided into those which are 'ideal', in which a definite strain occurs immediately on the application of a stress, and those which are non-ideal, showing a time lag. Ideal deformations may be Hockean (that is, directly proportional to stress) or non-Hookean. By definition, ideal deformations are completely recoverable, but non-ideal deformations may be either completely or incompletely recoverable. The latter give rise to visco-elastic deformations corresponding to systems which show no yield-value, that is, the smallest applied stresses produce a deformation.

Flow may be viscous (no yield-value) or plastic (yield-value). Here Dr. Treloar stressed the fact that postulating such categories does not imply
The table should also be extended in another dimension to allow for the fact that, for example, under constant stress, the rate of deformation of many materials accelerates (thixotropy, false body, etc.) or decelerates (work-hardening, dilatancy). Detailed subdivision of these properties has not yet been attempted by the committee but, so that the importance of such properties should not be forgotten, all categories for which such behaviour is not precluded by definition are included in a hatched 'frame'.

Dr. Treloar concluded by expressing the hope that mombers would criticize this table freely both with respect to suggested alterations and additions.

Dr. G. W. Scott Blair, honorary secretary of the Club, said that he thought members ought to know how much the committee owed to Dr. Treloar for his contribution to the preparation of the table. $\mathrm{He}$ had only one other point to make, namely, that those who worked in specialized fields would doubtless find the table still inadequate in the special 
parts which concerned them. For the present, a number of phenomena which are known to exist have been omitted since they are not of great practical importance, and it is hoped for the present to keep the table simple. Thus, just as rate of deformation can increase or decrease at constant stress, so the partial differential of strain with respect to stress can like. wise increase or decrease with stress considered at any given time. This would necessitate a second frame and a further subdivision of the 'non-ideal' category, but the committee believes that this is best not included for the present.

Mr. van Someren suggested that it would be helpful if each category could be considered in turn, and suggestions offered by members (not for inclusion in the table) of materials which would, under specified conditions, approximate in their behaviour to the category in question. This was done, though in some cases the suggestions were tentative.

There was some discussion on the meaning of 'recoverable' as applied to deformations. Dr. Scott Blair pointed out that the committee meant by 'recoverable' that the energy causing the recovery must be part of that originally applied and stored potentially, whether the recovery is spontaneous or initiated by some outside agent such as heat, mechanical or supersonic vibration, etc. Some members felt that 'recoverable' should be subdivided into 'spontaneously recoverable' and 'recoverable by activation by some external agent'. No final decision was reached and the committee was asked to consider the matter further.

A member raised the question of 'seepage' when the composition of a material is altered as it is de. formed, and Mr. J. C. Gage pressed the point that many complex properties such as thixotropy, false body, dilatancy, etc., have not yet been dealt with in the classification. Dr. Scott Blair said that the committee was grateful for such comments and would bear them in mind, and Dr. V. G. W. Harrison asked that members should express freely any views they might hold, especially about any important omissions in the table.

Mr. van Someren pointed out that, at the top of the table, elastic deformations appear on the left and flow ('liquid') properties on the right; whereas at the bottom, the plasto-elastic deformations, implying yield-values, a property of solids, are now on the right, and the visco-elastic on the left. The committee might consider some slight modification of the table by which this apparent anomaly might be avoided.

Mr. Maunder Foster stressed the importance of the adhesive properties of many materials, and gave some account of the significance of this group of rheological properties in the boot and shoe industry.

Dr. T. L. Ibbs, who had presided during the meeting, concluded by saying that, as a non-rheologist, he appreciated the simplicity of the table. It was easily understood by those who were not highly specialized in rheology, and he, for one, hoped that this simplicity might be maintained.

Following the discussion, it was unanimously resolved to accept the table of classification as a basis for further discussion.

Dr. G. W. Scott Blair then read a paper on "Rheological Developments Past and Present", illustrated by lantern slides. A number of members also showed apparatus and materials of rheological interest.

G. W. ScotT Bliair.

\section{INVESTIGATIONS ON LIGHTNING IN NIGERIA}

TN a recent paper entitled "The Measurement of Lightning Voltages and Currents in Nigeria, Part 2, 1938-1939", read in London before the Institution of Electrical Engineers, F. R. Perry, G. H. Webster and P. W. Baguley record the details of an investigation on the magnitude of lightning voltages and currents on Nigerian high-tension transmission lines, and continue an earlier work commenced by one of the authors. Three-phase oscillographic and klydonographic records of voltages were obtained, together with magnetic link measurements of currents on pole and lattice-mast structures. In addition, continuous records have been obtained of the interchange of current between earth and atmosphere during weather disturbances. The investigation resulted in a large amount of data for statistical analysis, and the results derived are briefly summarized below.

Surges of all types were analysed and resulted in wide variety of wave shapes, having wave-front values varying from 0.2 to 65 microsec., and wave. tail values from 0.9 to 93 microsec., the lowest values being generally associated with flash-over conditions. In general, the ratio between the length of wave tail to length of wave front covered a limited range of $1 \cdot 2 / 1$ to $4 / 1$, higher values being obtained on occasions, these ratios being practically independent of the wave front and tail magnitudes. These values were, for example, smaller than for the 1/50 microsec. wave usually adopted as the test standard.

A number of records of direct stroke voltages were also obtained, most of these being associated with power system faults caused by line insulation flashover. In such instances, the complete sequence of events was not always apparent, but it has been found that the recording of voltages on all three conductors is preferable to single-phase recording, a more complete history of the phenomenon being obtained. In two cases, it was found possible to obtain wave-shape records of the constituent strokes of multiple-stroke flashes, resulting in evidence of the family resemblance of such strokes. Certain aspects of the oscillographic records of lightning voltages were confirmed by klydonograms, the data obtained from the latter, however, being less accurate, and, in general, not so complete.

Current measurements were made in the case of direct line strokes, the line in this instance being supported by tubular steel poles, and in addition, the current distribution in the members of a lattice structure was obtained; of these, one set of such measurements involving eighteen lattice masts still awaits analysis.

Pronounced double pulses were obtained in certain oscillographic records of lightning voltages, and the suggestion is made that these are dependent on the current pulses in the main return lightning stroke, as was the opinion of Malan and Collens. Many of the observations made at Kew have been confirmed by observations made by means of a point-discharge recorder, certain differences relating to storm activity being simultaneously revealed with a contrast between temperate and tropical zone storm conditions. It was apparent from these records that a more complicated form of a Simpson-Robinson storm model could advantageously be applied to the task of explaining point-discharge records of lightning phenomena.

Following upon an analysis and discussion of the 